\title{
AJUSTE DE EQUAÇÕES ALOMÉTRICAS PARA Araucaria angustifolia (Bert.) O. Ktze. UTILIZANDO ANÁLISE DE COMPONENTES PRINCIPAIS CONJUNTAMENTE COM ANÁLISE DE REGRESSÃO
}

\author{
Rafaelo Balbinot ${ }^{1}$, Henrique Soares Koehler ${ }^{2}$, Luciano Farinha Watzlawick ${ }^{3}$, \\ Edemilson Alexandre Marcene ${ }^{4}$ \\ ${ }^{1}$ Eng. Florestal, M.Sc., Doutorando em Engenharia Florestal, UFPR, Curitiba, PR, Brasil - rbalbinot@yahoo.com.br \\ ${ }^{2}$ Eng. Florestal, Dr., Depto. de Fitotecnia e Fitossanitarismo, UFPR, Curitiba, PR, Brasil - koehler@ufpr.br \\ ${ }^{3}$ Eng. Florestal, Dr., Depto. de Agronomia, UNICENTRO, Irati, PR, Brasil - farinha@irati.unicentro.br \\ ${ }^{4}$ Acadêmico de Engenharia Florestal, UFPR, Curitiba, PR, Brasil - edemilson@ufpr.br
}

Recebido para publicação: 01/07/2008 - Aceito para publicação: 04/10/2008

\begin{abstract}
Resumo
O presente trabalho teve por objetivo a seleção de variáveis para estimativas do peso verde do fuste (pvf), peso verde de acículas (pva) e peso verde de galhos vivos (pvgv), por meio de Análise de Componentes Principais (ACP), em árvores nativas e plantadas de Araucaria angustifolia na região sul do estado do Paraná. Os dados analisados são provenientes de 15 árvores de diversas idades (29 a 33 anos), tendo sido avaliadas as variáveis diâmetro à altura do peito, altura total (h), altura comercial definida no ponto de inversão morfológica (hc), pvf, pva e pvgv. Como variáveis independentes (x), foram utilizados: dap, dap ${ }^{2}, \log d a p, h$, dap ${ }^{2} h, \operatorname{logh}$, hc, dap² hc e loghc, e como variáveis dependentes, pvf, pva e pvgv. Após o preparo dos dados para todas as relações dimensionais, foi gerada a matriz correlação, por meio da planilha Excel 2000, e posteriormente foi feita a ACP, utilizando-se o software Statgraphics 5.0. Concluiu-se que a utilização da ACP em conjunto com a Análise de Regressão apresenta grandes possibilidades para o ajuste de equações alométricas para Araucaria angustifolia, bem como para outras espécies florestais, sugerindo-se que, para a melhoria dos ajustes proceda-se à obtenção de um número maior de determinações de biomassa em outros indivíduos de Araucaria angustifolia.

Palavras-chave: Biomassa; araucária; peso verde.
\end{abstract}

\begin{abstract}
Fitting alometric equations for Araucaria angustifolia (Bert.) O. Ktze. using principal components analysis and linear regression. The main objective of the present research was to select variables for fitting alometric equations in order to estimate stem fresh weight (sfw), needles fresh weight (nfw) and branches fresh weight (BFW), through Principal Components Analysis, for native and planted Araucaria angustifolia trees, in the southern regions of Paraná State, Brazil. Data from 15 trees of several ages ( 29 to 33 years) being evaluated the following variables: dbh (diameter at breast height), $\mathrm{h}$ (total height) hc (commercial height), sfw, nfw and bfw. As independent variables (x) they were used dbh, dbh ${ }^{2}, \log (\mathrm{dbh}), \mathrm{ht}, \mathrm{dbh}{ }^{2} \mathrm{ht}, \log (\mathrm{h}), \mathrm{hc}, \mathrm{dbh}^{2} \mathrm{hc}$ and $\log (\mathrm{hc})$ and as dependent variables sfw, $\mathrm{nfw}$, bfw. Once data were computed for all variable combinations, a correlation matrix was generated by Excel 2000 and Principal Components Analysis was made by Statgraphics 5.0. The study leaded to the conclusion that the use of Principal Components Analysis together with Regression Analysis showed a great potential for fitting alometric equations for Araucaria angustifolia, as well as for other wood species. A greater number of tree measurements for biomass variables is advisable, to improve the fitting of biomass equations for Araucaria angustifolia.

Keywords: Biomass; araucaria; fresh weight.
\end{abstract}

\section{INTRODUÇÃO}

A quantificação da biomassa de uma árvore pode ser feita por dois métodos: direto e indireto. O método direto implica a derrubada da árvore para a determinação exata de seu peso e medidas. As determinações de biomassa em árvores utilizando o método direto são imprescindíveis para um primeiro 
conhecimento das características da espécie de interesse. Porém, tão logo se obtenha um número significativo de determinações, podemos lançar mão dos métodos indiretos, tais como os modelos de regressão. Utilizando esse método, é possível abranger grandes extensões florestais e reduzir significativamente a magnitude dos recursos financeiros necessários para esse tipo de trabalho.

Segundo Salati (1994), os métodos indiretos são utilizados para se estimar a biomassa de áreas florestais de grande extensão e, dependendo das informações disponíveis, são usadas relações empíricas entre a biomassa e algumas outras variáveis, determinando-se assim o valor da biomassa seca por hectare, para então ser feita uma extrapolação para a área total considerada. $\mathrm{O}$ autor ainda comenta que as variáveis comumente disponíveis nos inventários florestais são o diâmetro à altura do peito, a altura e o volume, as quais são relacionados de alguma forma com a biomassa.

Ogawa; Kira (1977) relatam que estudos sobre biomassa florestal têm principalmente envolvido o uso extensivo de alometria entre as diferentes dimensões de uma árvore em conjunto com determinações por meio da técnica de dissecação. Segundo Koehler, Watzlawick e Kirchner (2002), de forma geral têm-se duas variáveis, $\mathbf{y}$ e $\mathbf{x}$, tais que, quando representadas em um gráfico com escala logarítmica dupla, produzem uma linha reta, que pode ser expressa por $\mathrm{y}=\beta \mathrm{x}^{\alpha}$.

Nessa equação, $\beta$ e $\alpha$ são constantes específicas, sendo x a variável dependente, comumente representada pelo dap ou pela variável dap ${ }^{2} h$, e y o peso da biomassa.

Essa relação é universal e geralmente válida para qualquer relação entre as frações de biomassa, podendo ser usada para estimativa da biomassa do fuste, de galhos e de outras porções da árvore, porém com relações de dependência menos estáveis, alterando-se com características intrínsecas da floresta (OGAWA; KIRA, 1977).

Os métodos indiretos obviamente não podem ser utilizados sem o ajuste e a calibragem prévia das equações e, portanto, devem ser empregados em conjunto com os métodos diretos. Para Sanquetta (2002), quando do ajuste de equações, deve-se ter sempre o cuidado de avaliar com objetividade as melhores equações, por meio das estatísticas indicadoras de qualidade de ajuste $\left(\mathrm{s}_{\mathrm{yx}} \%\right.$ e $\left.\mathrm{r}^{2}\right)$. Higuchi e Carvalho Júnior (1994) indicam que o ideal é ter um modelo matemático para cada tipo florestal e para cada sítio, sendo que para o caso de estudos de mudanças climáticas a prioridade deve ser dada às áreas mais sujeitas às intervenções.

Contudo, geralmente, esses modelos apresentam limitações, que são ocasionadas pelo desconhecimento das relações alométricas (biológicas) entre as diversas variáveis, tais como diâmetro à altura do peito (dap), altura total (ht), altura comercial (hc), peso verde de galhos vivos (pvgv), peso de acículas (pva) e peso verde do fuste (pvf).

Segundo Vanclay (1994), as equações usadas para estimar biomassa são empíricas, pois descrevem o comportamento da variável sem explicar os fenômenos, o que não significa que sejam incapazes de fornecer estimativas biologicamente realistas dentro da amplitude de condições possíveis.

Portanto, a seleção das variáveis a serem testadas nos modelos e suas possíveis relações com a variável dependente e a amplitude dos dados amostrados são de fundamental importância para o sucesso das estimativas. Esse processo pode tornar-se especialmente complicado à medida que aumenta a diversidade de espécies.

Sobre métodos de seleção de variáveis, Isebrands; Crow (1975) afirmam que a Análise de Componentes Principais (ACP) pode ser utilizada em conjunto com regressão múltipla para a seleção de variáveis no ajuste de equações. A ACP é um procedimento analítico para transformação de um grupo de variáveis em outro grupo de componentes dessas variáveis, que têm as seguintes propriedades: (1) possuem funções lineares com as variáveis originais; (2) são ortogonais, isto é, independentes uns dos outros; (3) possuem a variação total das variáveis originais (consequentemente, não são observadas perdas de informações em sua transformação); e (4) a variância associada ao componente decresce em ordem. Os componentes são extraídos na ordem do mais explicativo para o menos explicativo. Teoricamente, o número de componentes é sempre igual ao número de variáveis. Entretanto, alguns poucos componentes são responsáveis por grande parte da explicação total. O processamento da ACP pode ter partida na matriz de variâncias e covariâncias ou na matriz de correlação. Se a opção for pela matriz de correlação, é aconselhável estabelecer o limite mínimo de 1.0 unidade para a extração dos autovalores.

O objetivo principal da ACP é a obtenção, a partir de um conjunto de variáveis, de um pequeno número de combinações lineares (componentes principais) que retenham o máximo possível da 
informação contida nas variáveis originais. Frequentemente, um pequeno número de componentes pode ser usado em lugar das variáveis originais nas análises de regressões (ISEBRANDS; CROW, 1975).

Com base nessas informações, o objetivo deste trabalho foi selecionar variáveis para o ajuste de equações alométricas para peso verde do fuste com casca (pvf), peso verde de acículas (pva) e peso verde de galhos vivos (pvgv), utilizando a Análise de Componentes Principais e tendo como base determinações de biomassa feitas em árvores nativas e plantadas de Araucaria angustifolia (Bert.) O. Ktze. na região sul do estado do Paraná.

\section{MATERIAL E MÉTODOS}

A área de estudo está localizada no município de General Carneiro, entre as coordenadas $26^{\circ} 20^{\prime} 35^{\prime \prime}$ e $26^{\circ} 26^{\prime} 13^{\prime}$ 'S, e $51^{\circ} 19^{\prime} 49^{\prime \prime}$ e $51^{\circ} 25^{\prime} 29^{\prime}$ 'W, na região sul do estado do Paraná, Brasil.

A vegetação natural é a Floresta Ombrófila Mista ou Floresta com Araucária (IBGE, 1992), que cobria originalmente cerca de $200.000 \mathrm{~km}^{2}$ em todo o Brasil, ocorrendo nos estados do Paraná (40\% de sua superfície), de Santa Catarina, de Minas Gerais e do Rio de Janeiro (1\%) (CARVALHO, 1994).

Os dados são provenientes de 15 árvores com idades entre 29 e 33 anos. A coleta de dados foi feita em fevereiro de 2001, medindo-se nas árvores avaliadas a circunferência à altura do peito (cap), a altura total (h), a altura comercial (hc) definida no ponto de inversão morfológica, o peso verde do fuste (pvf), o peso verde das acículas (pva) e o peso verde de galhos vivos (pvgv). A dimensão altura de copa também foi coletada durante a fase de campo, porém não foi utilizada por ser uma variável de difícil obtenção, principalmente em florestas naturais.

Como variáveis independentes $\mathrm{x}$ foram utilizados o dap, também nas formas quadrática e logarítmica (dap ${ }^{2}$ e logdap), h e hc, bem como suas multiplicações pela forma quadrática de dap (dap ${ }^{2}$ e dap²hc). Como variáveis dependentes, foram considerados o pvf, o pva e o pvgv.

Após a compilação dos dados para todas as relações dimensionais, foi gerada a matriz correlação, utilizando-se a planilha Excel2000, sendo posteriormente feita a ACP utilizando-se o software Statgraphics 5.0. Como se optou pela geração da matriz correlação, foi delimitado o valor de uma unidade para a extração dos autovalores da ACP.

As equações foram analisadas quanto à qualidade do ajuste com base no coeficiente de determinação $\left(\mathrm{r}^{2}\right)$ ajustado, erro padrão da estimativa em percentagem $\left(\mathrm{s}_{\mathrm{yx}} \%\right)$, distribuição gráfica dos resíduos e a significancia do teste $t$ de Student ao nível de 5\% de probabilidade de erro.

\section{RESULTADOS E DISCUSSÃO}

A ACP, a partir da matriz correlação, foi realizada para nove variáveis utilizadas, gerando os resultados apresentados na tabela 1 , que apresenta os autovalores, na ordem do mais explicativo para o menos explicativo, que absorvem a maior variação dos dados originais.

Tabela 1. Componentes principais, em ordem decrescente, de acordo com porcentagem da variação que representam em relação à variação original dos dados.

Table 1. Principal components, in decreasing order, according to variation percentage that represent in relation to original data variation.

\begin{tabular}{lccc}
\hline Número do componente & Autovalores & Porcentagem da variação & Porcentagem acumulada \\
\hline 1 & 4,7942 & 53,269 & 53,269 \\
2 & 1,9111 & 21,234 & 74,503 \\
3 & 1,5681 & 17,423 & 91,927 \\
4 & 0,4068 & 4,520 & 96,446 \\
5 & 0,1518 & 1,686 & 98,132 \\
6 & 0,1028 & 1,142 & 99,275 \\
7 & 0,0646 & 0,714 & 99,989 \\
8 & 0,0010 & 0,011 & 100,00 \\
9 & 0,0000 & 0,000 & 100,00 \\
\hline
\end{tabular}


Tendo sido utilizada a matriz correlação, os componentes selecionados foram apenas aqueles que tiveram o número do autovalor maior ou igual a 1 . Neste caso, das nove componentes geradas, somente as componentes 1, 2 e 3 foram selecionadas, as quais, juntas, absorveram 91,93\% da variação dos dados originais.

Após a seleção das componentes 1, 2 e 3, foram gerados os autovetores, relacionados na tabela 2 , para as nove variáveis utilizadas neste trabalho.

Tabela 2. Autovetores obtidos para as nove variáveis originais testadas.

Table 2. Obtained eigenvectors for the nine original variables tested.

\begin{tabular}{lccc}
\hline Variável & Componente 1 & Componente 2 & Componente 3 \\
\hline dap & 0,233113 & 0,579782 & 0,210308 \\
dap & 0,418273 & 0,003909 & $-0,233158$ \\
logdap & $-0,287288$ & 0,400302 & 0,291213 \\
ht & 0,298999 & $-0,0599624$ & $-0,542727$ \\
dap$^{2} \mathrm{~h}$ & 0,444459 & $3,23774^{16}$ & $-0,148967$ \\
logh & $-0,298999$ & 0,059962 & $-0,552727$ \\
hc & 0,287288 & $-0,400302$ & 0,291213 \\
dap'hc & 0,418273 & $-0,003909$ & $-0,233158$ \\
loghc & 0,231130 & $-0,579782$ & 0,210308 \\
\hline
\end{tabular}

De acordo com a metodologia da ACP, foram selecionados os maiores valores, em módulo, em cada um dos componentes. Na componente 1, foram selecionados dois autovetores, pois essa componente é a que explica sozinha $53,27 \%$ da variação, correspondendo às variáveis dap ${ }^{2}$ e dap ${ }^{2} \mathrm{~h}$. Nas componentes $2 \mathrm{e} 3$, foram selecionados os autovalores referentes às variáveis dap e logh, respectivamente.

A seguir, para cada um dos compartimentos da árvore a serem modelados foram feitas análises de variância utilizando-se todas as nove variáveis. Posteriormente, de acordo com metodologia da ACP e da Análise de Regressão, foi refeita a análise de variância utilizando-se somente as variáveis selecionadas pelo maior valor absoluto do autovetor.

\section{Peso verde de acículas (pva)}

A análise de variância do ajuste da equação para obtenção de estimativas de pva, incluindo todas as variáveis analisadas, é apresentada na tabela 3.

Tabela 3. Análise de variância do ajuste da equação de regressão com nove variáveis independentes (x) para estimar o pva (y).

Table 3. Variance analysis of linear regression fitting with nine independent variables $(\mathrm{x})$ to estimate needles fresh weight (nfw) (y).

\begin{tabular}{lccccc}
\hline F.V. & G. L. & S. Q. & Q. M. & F & Probabilidade \\
\hline Regressão & 9 & 206945,3 & 22993,92 & 8,743 & 0,01396 \\
Resíduo & 5 & 13149,95 & 2629,99 & & \\
\hline Total & 14 & 220095,3 & & & \\
\hline
\end{tabular}

De acordo com o teste t Student, existe uma relação estatística altamente significativa entre as variáveis independentes utilizadas (dap, dap ${ }^{2}, \operatorname{logdap}, \mathrm{h}, \mathrm{hc}, \mathrm{dap}^{2} \mathrm{~h}$ e dap $\mathrm{p}^{2} \mathrm{hc}$ ) e a variável dependente (pva). O $\mathrm{r}^{2}$ de $83,27 \%$ também é bom, porém o $\mathrm{s}_{\mathrm{yx}} \%$ de 40,06\% demonstra que, mesmo com todas variáveis independentes, o modelo ainda é impreciso.

Prosseguindo, a partir dos valores absolutos dos autovetores dos componentes 1,2 e 3 que, unidos, explicaram $91,93 \%$ da variação total, as respectivas variáveis foram selecionadas para novo ajustamento para estimativa do pva, conforme tabela 4.

A equação ajustada foi: pva $=-772,936+(-24,8912$ dap $)+\left(-0,772121\right.$ dap $\left.^{2}\right)+(0,01901$ dap² $h)+$ $(9694,06 \operatorname{logh})$. O coeficiente de determinação ajustado foi satisfatório $(84,29 \%)$, porém o erro padrão da estimativa $(39,68 \%)$ permanece demasiado alto para possibilitar o uso dessa equação. 
Tabela 4. Análise de variância do ajuste da equação de regressão com quatro variáveis independentes $\mathrm{x}$ (dap, dap², dap² e logh) para estimativa do pva (y).

Table 4. Variance analysis of linear regression fitting with four independent variables $\mathrm{x}\left(\mathrm{dbh}, \mathrm{dbh}^{2}\right.$, $\mathrm{dbh}^{2} \mathrm{~h}$ and $\operatorname{logh}$ ) to estimate $\mathrm{nfw}(\mathrm{y})$.

\begin{tabular}{lccccc}
\hline F.V. & G. L. & S. Q. & Q. M. & F & Probabilidade \\
\hline Regressão & 4 & 195391,80 & 48847,95 & 19,774 & 0,000 \\
Resíduo & 10 & 24703,44 & 2470,34 & & \\
\hline Total & 14 & 220095,3 & & & \\
\hline
\end{tabular}

Por se tratarem de exemplares nativos de Araucaria angustifolia, eles apresentam uma grande variação no peso da copa em função, principalmente, da competição que enfrentam no dossel das copas da floresta, que, ao contrário de um povoamento, não tem suas condições controladas.

Peso verde de galhos vivos (pvgv)

A tabela 5 apresenta a análise de variância do ajuste para o pvgv com as nove variáveis utilizadas neste estudo.

Tabela 5. Análise de variância do ajuste da equação de regressão com nove variáveis independentes (x) para obtenção das estimativas do pvgv (y).

Table 5. Variance analysis of linear regression fitting with nine independent variables (x) to estimate bfw (y).

\begin{tabular}{lccccc}
\hline F.V. & G. L. & S. Q. & Q. M. & F & Probabilidade \\
\hline Regressão & 5 & 4094022,0 & 818804,4 & 26,951 & 0,000 \\
Resíduo & 9 & 273429,9 & 30381,1 & & \\
\hline Total & 14 & 4367452,0 & & & \\
\hline
\end{tabular}

$\mathrm{O}$ teste $\mathrm{t}$ Student mostrou haver relação estatística altamente significativa entre as variáveis independentes utilizadas (dap, dap ${ }^{2}$ e logdap, h e hc, dap ${ }^{2} \mathrm{~h}$ e dap ${ }^{2} \mathrm{hc}$ ) e a variável dependente (pvgv). O ajuste foi muito bom $\left(\mathrm{r}^{2}=90,26 \%\right)$, porém ainda faltou precisão no modelo $\left(\mathrm{s}_{\mathrm{yx}} 42,05 \%\right)$.

Da mesma forma, selecionando-se as quatro variáveis de acordo com os valores absolutos dos autovetores dos componentes 1,2 e 3 , foi realizada a análise de variância do ajuste da equação, apresentada na tabela 6 .

Tabela 6. Análise de variância do ajuste da equação de regressão com as quatro variáveis independentes $\mathrm{x}$ (dap, dap², dap² e logh), para a obtenção das estimativas do pvgv (y).

Table 6. Variance analysis of linear regression fitting with four independent variables $\mathrm{x}\left(\mathrm{dbh}, \mathrm{dbh}^{2}\right.$, $\mathrm{dbh}^{2} \mathrm{~h}$ and $\operatorname{logh}$ ) to estimate bfw (y).

\begin{tabular}{lccccc}
\hline F.V. & G. L. & S. Q. & Q. M. & F & Probabilidade \\
\hline Regressão & 4 & 4054187,0 & 1013547,0 & 32,354 & 0,000 \\
Resíduo & 10 & 313265,2 & 31326,5 & & \\
\hline Total & 14 & 4367452,0 & & & \\
\hline
\end{tabular}

Equação ajustada para pvgv: $-2668,97+(-107,215$ dap $)+\left(2,813432\right.$ dap $\left.^{2}\right)+(0,06222$ dap²h $)+$ $(3627,675 \mathrm{logh})$. Os resultados do coeficiente de determinação ajustado $(89,96 \%)$ e do erro padrão da estimativa $(42,21 \%)$ se mostraram semelhantes aos do ajuste para o pva, provavelmente pelo mesmo motivo da competição entre as copas.

\section{Peso verde de fuste (pvf)}

A tabela 7 apresenta os resultados da análise de variância para o ajuste da equação que estima o pvf para as nove variáveis utilizadas neste estudo.

$\mathrm{O}$ teste $\mathrm{t}$ Student mostrou haver relação estatística altamente significativa entre as variáveis independentes utilizadas (dap, dap ${ }^{2}, \operatorname{logdap}, \mathrm{h}, \mathrm{hc}, \mathrm{dap}^{2} \mathrm{~h}$ e dap ${ }^{2} \mathrm{hc}$ ) e a variável dependente (pvf). O pvf foi a variável que apresentou o melhor coeficiente de determinação ajustado $(99,76 \%)$ e com o menor erro padrão da estimativa $(8 \%)$. 
Tabela 7. Análise de variância do ajuste da equação de regressão com nove variáveis independentes (x) para a obtenção das estimativas do pvf (y).

Table 7. Variance analysis of linear regression fitting with nine independent variables (x) to estimate sfw (y).

\begin{tabular}{lccccc}
\hline F.V. & G. L. & S. Q. & Q. M. & F & Probabilidade \\
\hline Regressão & 9 & $1,18 \mathrm{E}+08$ & 13080352,0 & 656,693 & 0,000 \\
Resíduo & 5 & 99592,58 & 19918,5 & & \\
\hline Total & 14 & $1,18 \mathrm{E}+08$ & & & \\
\hline
\end{tabular}

A partir das quatro variávies selecionadas pela ACP, foi feito o ajuste ajuste do modelo, cuja análise de variância é mostrada na tabela 8.

Tabela 8. Análise de variância do ajuste da equação de regressão com as quatro variáveis independentes $\mathrm{x}$ (dap, dap ${ }^{2}$, dap² e logh) para a obtenção das estimativas do pvf (y).

Table 8. Variance analysis of linear regression fitting with four independent variables $\mathrm{x}\left(\mathrm{dbh}, \mathrm{dbh}^{2}\right.$, $\mathrm{dbh}^{2} \mathrm{~h}$ and $\operatorname{logh}$ ) to estimate sfw (y).

\begin{tabular}{lccccc}
\hline F.V. & G. L. & S. Q. & Q. M. & F & Probabilidade \\
\hline Regressão & 4 & $1,18 \mathrm{E}+08$ & 29420170,0 & 2070,663 & 0,000 \\
Resíduo & 10 & 142080,9 & 14208,1 & & \\
\hline Total & 14 & $1,18 \mathrm{E}+08$ & & & \\
\hline
\end{tabular}

A equação ajustada foi: $p v f=-929,564+(-43,2438$ dap $)+\left(1,772849\right.$ dap $\left.^{2}\right)+(-0,01332$ dap² $h)+$ $(1220,484 \operatorname{logh})$. O ajuste da equação que estima o pvf para Araucaria angustifolia, com coeficiente de determinação ajustado de $99,83 \%$ e erro padrão da estimativa de $6,76 \%$, foi o que se mostrou possível de ser utilizado para a obtenção de estimativas de biomassa verde do fuste, a partir de variáveis de fácil obtenção, como dap e h, seguindo-se o método de ACP. Outro fator que demonstrou a alta qualidade do ajuste foi a alta significância estatística entre as variáveis independentes e a variável dependente, com $\mathrm{F}$ calculado muito maior que o tabelado.

No tocante às correlações para araucária, pôde-se observar que as variáveis analisadas neste trabalho são altamente correlacionadas entre si, porém ainda carecem de precisão no caso das estimativas para pva e pvgv. Provavelmente, esse problema poderá ser sanado com o aumento do número de amostras de árvores nativas. Em plantações de araucária, as variáveis parecem ter correlação ainda melhor. Em estudo utilizando o modelo b0 + b1 dap + b2 dap ${ }^{2}+$ b3 dap ${ }^{2}$, Sanquetta et al. (2003) obtiveram os seguintes resultados para $\mathrm{r}^{2 \%}$ e $\mathrm{s}_{\mathrm{yx}} \%$ : pvf $=99,9$ e 4,6, pva $=99,7$ e 11,2, e pvgv $=99,9$ e 7,3. Essas correlações sugerem que estimar biomassa a partir de variáveis de inventários convencionais, como as utilizadas neste trabalho, é uma tarefa viável, particularmente para a porção fuste. A inclusão de variáveis de copa, tais como diâmetro e comprimento, provavelmente devem reduzir os erros percentuais dos modelos, porém não foram utilizadas por serem de difícil obtenção, principalmente em florestas naturais.

O prosseguimento dos estudos sobre o ajuste de equações alométricas para as espécies florestais brasileiras de interesse silvicultural facilitará, em futuro breve, as estimativas de biomassa e carbono fixado realizadas para projetos do Mecanismo de Deseonvolvimento Limpo, do Protocolo de Quioto, direcionados para florestamentos, reflorestamentos e florestas naturais.

\section{CONCLUSÕES}

- O uso da ACP em conjunto com Análise de Regressão apresenta grandes possibilidades no desenvolvimento de equações alométricas para Araucaria angustifolia.

- Para melhoria dos ajustes, é necessária a obtenção de um número maior de determinações de biomassa em outros indivíduos de Araucaria angustifolia.

- Os resultados dos ajustes para pva e pvgv não se mostraram satisfatórios para serem utilizados em estimativas do peso desses componentes para a espécie Araucaria angustifolia.

- A estimativa gerada a partir da equação pvf $=-929,564+(-43,244$ dap $)+\left(1,773\right.$ dap $\left.^{2}\right)+(-0,013$ dap$\left.^{2} h\right)+(1220,484 \operatorname{logh})$ mostrou-se segura. 


\section{REFERÊNCIAS}

ANDRAE, F. Effects of nutrient accumulation by aspen, spruce and pine on soil properties. Soil Science Society American Journal, Madison, v. 46, p. 853-861, 1982.

BALBINOT, R.; SCHUMACHER, M. V.; IENSEN, E. A. M.; HERNANDES, J. I. Determinação da quantidade de carbono orgânico na biomassa aérea e subterrânea de um povoamento de Pinus taeda, com 10 anos de Idade, na Região de Cambará do Sul - RS. In: CONGRESSO FLORESTAL ESTADUAL DE NOVA PRATA - RS, 8., 2001, Nova Prata. Anais... Nova Prata: [s.n.], 2001. p. 454-457.

CARVALHO, P. E. R. Espécies florestais brasileiras: recomendações silviculturais, potencialidades e uso da madeira. Colombo: EMBRAPA-CNPF; Brasília, DF: EMBRAPA-SPI, 1994. 640 p.

GOLLEY, F. B.; MCGINNIS, J. T.; CLEMENTS, R. G.; CHILD, G. I.; DUEVER, M. J. Ciclagem de minerais em um ecossistema de floresta tropical úmida. São Paulo: USP, 1978. 256 p.

HIGUCHI, N.; CARVALHO JÚNIOR, J. A. Fitomassa e conteúdo de carbono de espécies arbóreas da Amazônia. In: SEMINÁRIO EMISSÃO X SEQÜESTRO DE $\mathrm{CO}_{2}$ - uma nova oportunidade de negócios para o Brasil, 1994. Rio de Janeiro. Anais... Rio de Janeiro: Companhia Vale do Rio Doce. p. 125-145.

ISEBRANDS, J. G.; CROW, T. R. Introduction to uses and interpretation of principal components analysis in forest biology. St. Paul: USDA Forest Service, 1975. 19 p.

KOEHLER, E. S.; WATZLAWICK, L. F.; KIRCHNER, F. F. Fontes e níveis de erros nas estimativas do potencial de fixação de carbono. In: As Florestas e o carbono. SANQUETTA, C. R. (Ed.). Curitiba, 2002. p. 251-264.

OGAWA, H.; KIRA, T. Methods of estimating forest biomass. In: Primary productivity of Japanese forests - Productivity of terrestrial communities. Journal of Integrative Plant Biology, Beijing, v. 16, 1977. p. 15-24

SALATI, E. Emissão x seqüestro de $\mathrm{CO}_{2}$ - uma nova oportunidade de negócios para o Brasil. In: Seminário emissão x seqüestro de $\mathbf{C O}_{2}$ - uma nova oportunidade de negócios para o Brasil. Rio de Janeiro: Companhia Vale do Rio Doce, 1994. p. 15-37.

SANQUETTA, C. R. Métodos de determinação de biomassa florestal. In: As Florestas e o carbono. SANQUETTA, C. R. (Ed.). Curitiba, 2002. p. 119-140.

SANQUETTA, C. R.; WATZLAWICK, L. F.; SCHUMACHER, M. V.; MELlO, A. A. Relações individuais de biomassa e conteúdo de carbono em plantações de Araucária angustifolia e Pinus taeda no sul do estado do Paraná, Brasil. Revista Acadêmica: ciências agrárias e ambientais, Curitiba, v. 1, n. 3, p. 33-40, jul./set. 2003.

WATZLAWICK, L. F.; KIRCHNER, F. F.; SANQUETTA, C. R.; SCHUMACHER, M. V. Fixação de Carbono em Floresta Ombrófila Mista em diferentes estágios de regeneração. In: As Florestas e o carbono. SANQUETTA, C. R. (Ed.). Curitiba, 2002. p. 153-190. 